\title{
Consideration of lightning current along a tall structure in the presence of horizontal arrangement grounding
}

\begin{abstract}
The evaluation of the behaviour of lightning current along a tall structure is becoming an important issue when considering the lightning protection equipment required along a tall structure. This behaviour is affected by the element of the ground reflection factor which has a correlation between the impedance of the ground and the tower. The impedance of the ground is considered for a horizontal grounding arrangement while the impedance of the tower is assumed to be constant. Hence, the ground reflection factor can be given a variable value, whereas most of the existing literature assumes a constant value. When a constant value is entered into a calculation field, an inaccurate result is computed.In this paper, the lightning current along a tall structure under varying values of ground reflection factor and including the effect of the presence of a horizontal grounding arrangement has been considered and the results discussed accordingly. Modelling of the lightning return stroke current along a tall structure was undertaken by entering the calculated variable value of the ground reflection factor. The results indicated that a variable value of the ground reflection factor causes different peak values of the current and wave shape of the lightning current for different positions along a tall structure.
\end{abstract}

Keyword: Grounding system; Lightning; Soil resistivity; Tall structures 